\title{
Temporal shifts in the skin microbiome associated with disease flares and treatment in children with atopic dermatitis
}

\author{
Heidi H. Kong, ${ }^{1,8}$ Julia Oh, ${ }^{2}$ Clay Deming, ${ }^{2}$ Sean Conlan, ${ }^{2}$ Elizabeth A. Grice, ${ }^{2}$ \\ Melony A. Beatson, ${ }^{1}$ Effie Nomicos, ${ }^{1}$ Eric C. Polley, ${ }^{3}$ Hirsh D. Komarow, ${ }^{4}$ NISC Comparative \\ Sequence Program, ${ }^{5,7}$ Patrick R. Murray, ${ }^{6}$ Maria L. Turner, ${ }^{1}$ and Julia A. Segre ${ }^{2,8}$ \\ ${ }^{7}$ Dermatology Branch, Center for Cancer Research, National Cancer Institute, NIH, Bethesda, Maryland 20892, USA; ${ }^{2}$ Genetics and \\ Molecular Biology Branch, National Human Genome Research Institute, NIH, Bethesda, Maryland 20892, USA; ${ }^{3}$ Biometric Research \\ Branch, Division of Cancer Treatment and Diagnosis, National Cancer Institute, NIH, Bethesda, Maryland 20892, USA; ${ }^{4}$ Laboratory \\ of Allergic Diseases, National Institute of Allergy and Infectious Diseases, NIH, Bethesda, Maryland 20892, USA; ${ }^{5}$ NIH Intramural \\ Sequencing Center, National Human Genome Research Institute, NIH, Bethesda, Maryland 20892, USA; ${ }^{6}$ Microbiology Service, \\ Department of Laboratory Medicine, Clinical Center, NIH, Bethesda, Maryland 20892, USA
}

\begin{abstract}
Atopic dermatitis (AD) has long been associated with Staphylococcus aureus skin colonization or infection and is typically managed with regimens that include antimicrobial therapies. However, the role of microbial communities in the pathogenesis of $A D$ is incompletely characterized. To assess the relationship between skin microbiota and disease progression, $16 \mathrm{~S}$ ribosomal RNA bacterial gene sequencing was performed on DNA obtained directly from serial skin sampling of children with $A D$. The composition of bacterial communities was analyzed during AD disease states to identify characteristics associated with $A D$ flares and improvement post-treatment. We found that microbial community structures at sites of disease predilection were dramatically different in $A D$ patients compared with controls. Microbial diversity during $A D$ flares was dependent on the presence or absence of recent AD treatments, with even intermittent treatment linked to greater bacterial diversity than no recent treatment. Treatment-associated changes in skin bacterial diversity suggest that $A D$ treatments diversify skin bacteria preceding improvements in disease activity. In AD, the proportion of Staphylococcus sequences, particularly S. aureus, was greater during disease flares than at baseline or post-treatment, and correlated with worsened disease severity. Representation of the skin commensal S. epidermidis also significantly increased during flares. Increases in Streptococcus, Propionibacterium, and Corynebacterium species were observed following therapy. These findings reveal linkages between microbial communities and inflammatory diseases such as $A D$, and demonstrate that as compared with culture-based studies, higher resolution examination of microbiota associated with human disease provides novel insights into global shifts of bacteria relevant to disease progression and treatment.
\end{abstract}

[Supplemental material is available for this article.]

Atopic dermatitis (AD, "eczema," OMIM 603165) is a chronic, relapsing, intensely pruritic inflammatory skin disorder that can be successfully treated with varying combinations of topical or systemic antibiotics, corticosteroids, and dilute bleach baths (Huang et al. 2009). The prevalence of $\mathrm{AD}$ has more than doubled in industrialized countries with no clear cause (Asher et al. 2006; Shaw et al. 2011) and at a high cost (Bickers et al. 2006). More than half of children with moderate to severe $\mathrm{AD}$ develop allergic rhinitis and/or asthma, atopic disorders associated with significant morbidity and rare mortality. Genetic as well as environmental factors affect the expression of allergic diseases, including $\mathrm{AD}$ (von Mutius 2000; Cramer et al. 2010). Mutations in $F L G$, the gene encoding the skin barrier protein filaggrin, are associated with $\mathrm{AD}$, particu-

\footnotetext{
${ }^{7}$ A complete list of of the NISC Comparative Sequencing Program investigators appears at the end of this manuscript.

${ }^{8}$ Corresponding authors.

E-mail konghe@mail.nih.gov.

E-mail jsegre@mail.nih.gov.

Article published online before print. Article, supplemental material, and publication date are at http://www.genome.org/cgi/doi/10.1101/gr.131029.111.
}

larly in patients who subsequently develop asthma and/or allergic rhinitis, suggesting that epicutaneous sensitization may contribute to atopic disease (Palmer et al. 2006; Sandilands et al. 2007).

$\mathrm{AD}$ patients experience frequent cutaneous infections, and Staphylococcus aureus is commonly cultured from lesional and nonlesional AD skin (Leyden et al. 1974). Reduced antimicrobial peptide expression in the skin of $\mathrm{AD}$ patients may contribute to this susceptibility (Ong et al. 2002). While individual microbes causative of common AD skin infections have been studied, it is increasingly clear that individual microbes function within larger bacterial communities (Cogen et al. 2010b).

High-throughput DNA sequencing of the bacterial 16S rRNA gene has revealed a vastly greater bacterial diversity on healthy human skin than demonstrated by culture-based methods (Costello et al. 2009; Grice et al. 2009). The skin's topography and microenvironments are powerful determinants of microbial community structure at particular skin sites (Gao et al. 2007; Costello et al. 2009; Grice et al. 2009). AD preferentially involves the antecubital and popliteal regions, sites that harbor similar groups of organisms and share distinct compositions of microbial communities (Grice et al. 2009). These findings suggest that microbial communities 
might underlie or contribute to observed predilections of some dermatologic disorders for stereotypical sites.

To examine the role of the skin microbiome in $\mathrm{AD}$, we performed a skin microbiome study of $\mathrm{AD}$ disease states (baseline disease state, disease flare, and post-treatment for disease flare) in 12 pediatric patients with moderate-to-severe $\mathrm{AD}$ and 11 healthy controls, aged $2-15 \mathrm{yr}$ (Table 1; Supplemental Table S1). In this study, we demonstrate how different disease states, severity, and treatments relate to microbial dynamics in this common skin disorder. We report dramatic reductions in the skin microbial diversity during AD flares, which is restored with common AD therapies.

\section{Results}

\section{Characteristics of bacterial communities associated with $A D$}

To investigate the role of bacterial communities in $\mathrm{AD}$, we obtained skin samples from 12 children with moderate-to-severe $\mathrm{AD}$ and from 11 controls recruited to the NIH Clinical Center between August 2008 and July 2010. Disease severity was assessed at predetermined sampling timepoints: baseline, stable disease state (B); acute disease flare (F); and 10-14-d post-flare treatment (PF). Disease severity was assessed quantitatively with SCORAD (SCORing AD), a well-validated clinical assessment tool (Williams et al. 1994b; Kunz et al. 1997; Oranje et al. 2007). We obtained skin samples from the left and right antecubital (Ac) and popliteal creases (Pc) - sites of predilection for AD in this age group (Fig. 1A). We also sampled volar forearms (Vf) as control skin sites that are adjacent to, but not a classical site of disease predilection itself, and the nares $(\mathrm{N})$ as a reservoir for $S$. aureus colonization (Archer and Climo 2001; von Eiff et al. 2001). We sequenced skin samples from $36 \mathrm{AD}$ patient visits and 24 control visits for a total of 151,924 nearfull-length 16S ribosomal RNA (rRNA) genes (Supplemental Table S2). Study design is detailed in the Methods section.

Table 1. Clinical characteristics of the participants ${ }^{a}$

\begin{tabular}{|c|c|c|}
\hline Characteristic & $\begin{array}{l}\text { Atopic dermatitis } \\
\text { (AD) patients }\end{array}$ & $\begin{array}{c}\text { Age-matched } \\
\text { healthy controls }\end{array}$ \\
\hline Total subjects analyzed & $12^{\mathrm{a}}$ & 11 \\
\hline Age, mean (range) & 7.8 yr $(2-15)$ & 8.0 yr $(2-14)$ \\
\hline Male:female & $8: 4$ & $6: 5$ \\
\hline Baseline objective SCORAD ${ }^{\mathrm{b}, \mathrm{c}}$ & $21.8 \pm 3.1$ & N/A \\
\hline Flare objective SCORAD & $42.1 \pm 2.2$ & N/A \\
\hline Post-flare objective SCORAD & $18.1 \pm 3.0$ & N/A \\
\hline Asthma $^{\mathrm{d}}$ & 7 of 12 & 0 \\
\hline Allergic rhinitis ${ }^{d}$ & 8 of 12 & 0 \\
\hline Family history positive of $A D$ & 8 of 12 & 4 of 11 \\
\hline All nares cultures positive for S. aureus (\%) & 8 of 12 & 5 of 11 \\
\hline All nares cultures negative for S. aureus (\%) & 0 & 5 of 11 \\
\hline Eosinophils (\%) $)^{\mathrm{e}}$ & $8.43 \pm 1.66$ & $3.44 \pm 0.92$ \\
\hline
\end{tabular}

${ }^{a}$ Evaluated during at least one full cycle of baseline, flare, postflare. Four additional patients were analyzed at flare.

${ }^{b}$ Baseline characteristics of participants at initial visit. Plus-minus values are means $\pm S E$.

'SCORing atopic dermatitis.

${ }^{\mathrm{d}}$ As defined by International Study of Asthma and Allergies in Childhood questionnaire.

e Normal range for eosinophils is $0.8 \%-7.0 \%$.
Mean objective SCORAD scores designating AD disease severity were significantly elevated during flares $(42 \pm 2.2)$ as compared with baseline $\left(22 \pm 3.1, P<7.3 \times 10^{-4}\right)$ and postflare $(18 \pm 3.0, P<0.0038)$ (Fig. 1B). Categorization of baseline, flare, and postflare was based on patient clinical status and specified frequency of $\mathrm{AD}$ treatment administration (see Methods section for detailed classification criteria). Flare was defined by worsening disease irrespective of use of therapy $>24 \mathrm{~h}$ prior to sampling. We examined the recent treatment history prior to flares because we predicted that the timing of treatments in relation to sampling might influence the skin microbiome. We found that while no significant differences were detected in objective SCORAD scores $(P<0.22)$, flare timepoints could be grouped into patients that used (1) "no-treatment" $(n=7)$, defined as patients who reported no use of topical medications (corticosteroids, calcineurin inhibitors, or antibiotics) for $\geq 7 \mathrm{~d}$, and no ingestion of oral antibiotics $\geq 4 \mathrm{wk}$ prior to flare skin sampling, or (2) "intermittent-treatment" $(n=5)$, defined as patients who reported use of topical medications in the previous $7 \mathrm{~d}$ and/or ingestion of oral antibiotics in the previous $4 \mathrm{wk}$ before skin sampling (Table 2).

To investigate a possible relationship, we analyzed the association between $\mathrm{AD}$ disease severity and Shannon diversity, an ecological measure of microbial communities that considers: (1) richness, or the total number of bacterial types, and (2) evenness, or the relative proportion of these bacterial types. The antecubital and popliteal creases are considered sites of disease predilection and showed similar results. We averaged these sites per subject ("AcPc") for subsequent analyses, unless otherwise indicated, to avoid treating related samples from the same individual as biological replicates. The partial correlation between objective SCORAD and Shannon diversity of AcPc, adjusting for disease state, was significantly inversely correlated $\left(r=-0.57, P<3.6 \times 10^{-4}\right)$ (Fig. $2 \mathrm{~A})$, indicating that severe $\mathrm{AD}$ was associated with lower skin bacterial diversity at sites of disease predilection.

$\mathrm{AD}$ patients using no treatments during flares exhibited markedly reduced Shannon diversity in the antecubital creases as compared with intermittenttreatment use $(P<0.025)$ (Fig. 2B,C; Supplemental Table S3). Shannon diver- 
Table 2. Atopic dermatitis treatments prior to flare sampling

\begin{tabular}{|c|c|c|c|c|}
\hline & $\begin{array}{c}\text { Topical } \\
\text { medications }\end{array}$ & $\begin{array}{l}\text { Dilute } \\
\text { bleach } \\
\text { baths }\end{array}$ & $\begin{array}{c}\text { Systemic } \\
\text { antibiotics }\end{array}$ & $\begin{array}{c}\text { Shannon diversity } \\
\text { index (antecubital } \\
\text { crease at flare) }\end{array}$ \\
\hline $\begin{array}{l}\text { Time prior to sampling } \\
\text { (A) No treatment }{ }^{\mathrm{B}}\end{array}$ & $\leq 7 \mathrm{~d}$ & $\leq 7 \mathrm{~d}$ & $\leq 4 \mathrm{wk}$ & \\
\hline AD02 & - & - & - & low (0.76) \\
\hline AD03 & - & - & - & low $(0.11)$ \\
\hline AD04 & - & - & - & low $(0.44)$ \\
\hline AD05 & - & - & - & low (0.59) \\
\hline AD07 & - & - & - & low (0.35) \\
\hline AD10 & - & - & - & high (1.37) \\
\hline AD11 & - & - & - & low $(0.40)$ \\
\hline \multicolumn{5}{|l|}{ (B) Intermittent treatment $\mathrm{C}^{\mathrm{C}}$} \\
\hline AD01 & + & + & + & high (3.27) \\
\hline AD06 & + & - & - & high (3.08) \\
\hline AD08 & + & - & - & high (3.26) \\
\hline AD09 & + & - & - & high (2.38) \\
\hline \multirow{2}{*}{\multicolumn{5}{|c|}{ (C) Additional patient flares ${ }^{d}$}} \\
\hline & & & & \\
\hline AD13 & - & Incomplete data & & low $(0.60)$ \\
\hline AD14 & - & - & - & low (0.27) \\
\hline AD15-1st flare & - & - & - & low (0.63) \\
\hline AD15-2nd flare & + & + & - & high (1.65) \\
\hline AD16 & - & + & - & high (3.75) \\
\hline
\end{tabular}

${ }^{\text {a }}$ Topical medications include corticosteroids, antibiotics, and calcineurin inhibitors.

${ }^{\mathrm{b}}$ No treatment group $(n=7)$ represents $\mathrm{AD}$ patients who used topical medications $>7 \mathrm{~d}$ and/or oral antibiotics $>4$ wk prior to disease flare sampling.

Intermittent treatment group $(n=5)$ represents AD patients who used topical medications $\leq 7 \mathrm{~d}$ and/or oral antibiotics $\leq 4 \mathrm{wk}$ prior to disease flare sampling timepoint.

${ }^{\mathrm{d}}$ Additional AD patients $(n=4)$ sampled during flares. AD15 had two flare visits.

sity during intermittent-treatment flares was not significantly different from baseline, postflares, or controls. Similar trends were observed for popliteal creases, another site of disease predilection (Fig. 2C; Supplemental Table S3). These results suggest that cutaneous microbial diversity is associated with disease status in the context of recent treatment history, and that $\mathrm{AD}$ treatments can prevent or reverse the change in diversity associated with flares even before clinical improvement is seen. In contrast to the antecubital and popliteal creases, diversity in the nares as well as the volar forearm was relatively constant, demonstrating that changes in bacterial diversity were specific to sites of disease predilection (Fig. 2C; Supplemental Table S3).

To assess how distinct the skin microbiomes for each $\mathrm{AD}$ disease state and controls were from each other, we compared the level of similarity of bacterial community structures with the YueClayton theta similarity coefficient $(\theta)$ (Yue and Clayton 2005). The $\theta$ index takes into consideration the number of bacterial species present and their relative abundances in the two communities being compared (Yue and Clayton 2005; Schloss and Handelsman 2006) with $\theta=1$ indicating identical community structure and $\theta=0$ indicating absolute dissimilarity. AD patients within the no-treatment
A

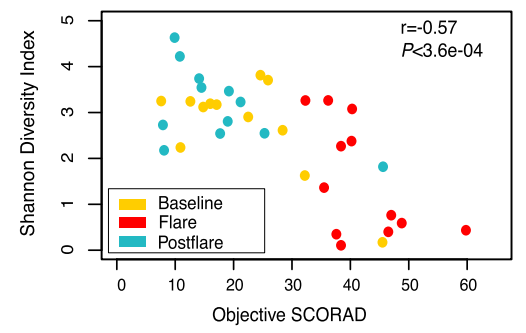

C

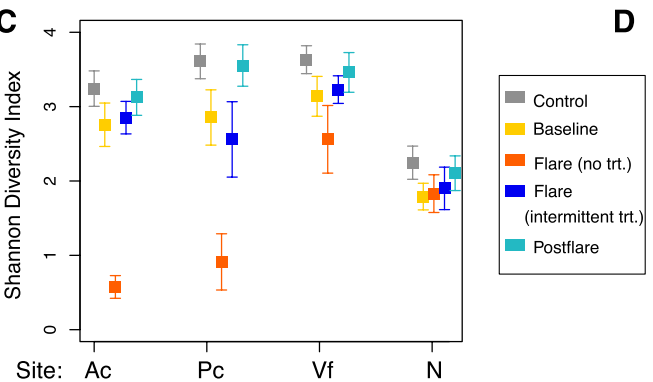

flare group $(\theta=0.91 \pm 0.029)$ were statistically significantly more similar to each other than baseline, intermittenttreatment flares, and postflare (Fig. 2D; Supplemental Fig. S1; Supplemental Table S4), far exceeding the reference point of interpersonal variation observed in the within-group $\theta$ for controls $(\theta=0.26 \pm$ $0.025, P<4.3 \times 10^{-10}$ ). Our results underscored that no-treatment flares were distinguishable from intermittent-treatment flares and likely reflected the natural disease course unaltered by recent medications.

To investigate whether these bacterial community shifts across timepoints were unique to the $\mathrm{AD}$ cohort, we calculated both Shannon and Theta indices across visits for the healthy controls, who were sampled at similar time intervals as AD patients (Supplemental Table S5). Mean changes in Shannon diversity between visits were significantly higher for no-treatment $\mathrm{AD}$ flare patients (baseline/ flare, and flare/postflare) as compared with healthy controls $(P<0.002$ and $P<$ 0.02 , respectively, for Ac and Pc). Similarly, mean theta values between visits were significantly lower in the no-treatment AD flare group than healthy controls in the Ac $(P<0.03)$. We conclude from this analysis that the skin bacterial community shifts across timepoints are significantly different in $\mathrm{AD}$ patients as compared with similarly sampled healthy controls.
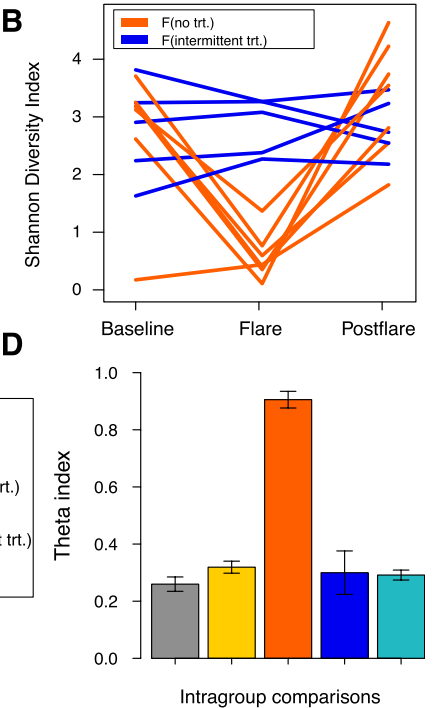

Figure 2. Microbial community-level statistics in the AD microbiome. (A) Relationship between objective SCORAD and Shannon diversity in the Ac of AD patients. Partial correlation (adjusting for disease state). (B) Longitudinal Shannon diversity trend in AD grouped by no-treatment (trt) and intermittent-trt flares $(n=12, \mathrm{AC})$. (C) Mean Shannon diversity \pm SEM in controls and all AD disease states (AC, PC, volar forearm $[V f]$, nares $[N])$. $(D)$ Mean theta $(\theta)$ similarity coefficients \pm SEM. Pairwise comparisons of community structure between individuals within a control or AD disease group. Bars represent average of all pairwise comparisons of community structure for control individuals to other controls, baselines to baselines, flares(no-trt)-flares(no-trt), flare(intermittent-trt)-flare(intermittent-trt), and postflares-postflares. 


\section{Selective bacterial shifts and $A D$ disease states}

To identify bacteria contributing to the reduction in $\mathrm{AD}$ microbial diversity, we classified the phylum, order, and genus of the sequences (Supplemental Fig. S2; Supplemental Table S6) at each site. To determine the similarity of the bacterial communities between sites at a taxonomic level, we first performed cross-site correlations of the abundances of each taxa at the phylum-order level (Supplemental Fig. S3). In AD patients, the antecubital and popliteal creases were highly similar (partial correlation adjusting for disease state $r=0.80)$, exceeding the similarity of these sites in controls ( $r=$ 0.68 , Fisher r-to-z transformation, $P<0.0002$ ). The similarity between the antecubital and popliteal creases relates to the observed localization of $\mathrm{AD}$ in these sites and further justifies combining these sites for analysis. Antecubital creases were similar to the adjacent volar forearm in both $\mathrm{AD}$ and control individuals $(r=0.79$ and 0.81 , respectively), suggesting that the typically unaffected skin of the volar forearm significantly correlated with sites of disease predilection. In general, the nares taxa were poorly correlated with the skin sites for all individuals.

Examining the relative abundance of specific taxa at each timepoint, we observed that the antecubital and popliteal creases were strikingly dominated by the genus Staphylococcus. The notreatment flares had the highest abundance of bacterial $16 \mathrm{~S}$ genes classified as Staphylococcus at $90 \pm 1.6 \%$ as compared with other $\mathrm{AD}$ patients and controls $(35 \pm 2.2 \%, P<0.013$ [baseline]; $31 \pm$ 5.0\%, $P<0.050$ [intermittent-treatment flares]; $20 \pm 1.3 \%, P<$ $2.0 \times 10^{-4}$ [postflare]; $16 \pm 1.8 \%, P<6.2 \times 10^{-4}$ [controls]) (Fig. 3A; Supplemental Table S7).

The genus Staphylococcus includes several bacterial species relevant to clinical disease, including $S$. epidermidis as a skin commensal (Wisplinghoff et al. 2004; Hidron et al. 2008) and S. aureus as a pathogen with a known association with AD. To distinguish among Staphylococcal species, we generated a custom 16S rRNA sequence database to uniquely classify the Staphylococcal sequences (Fig. 3B). The proportion of $S$. aureus in the no-treatment flares was $65 \pm 3.5 \%$, statistically significantly higher than baseline, postflare, and controls (Fig. 4A; Supplemental Table S7). The proportion of $S$. aureus in the intermittent-treatment flares $(15 \pm$ $2.5 \%)$ was significantly higher than controls $(P<0.020)$, but not
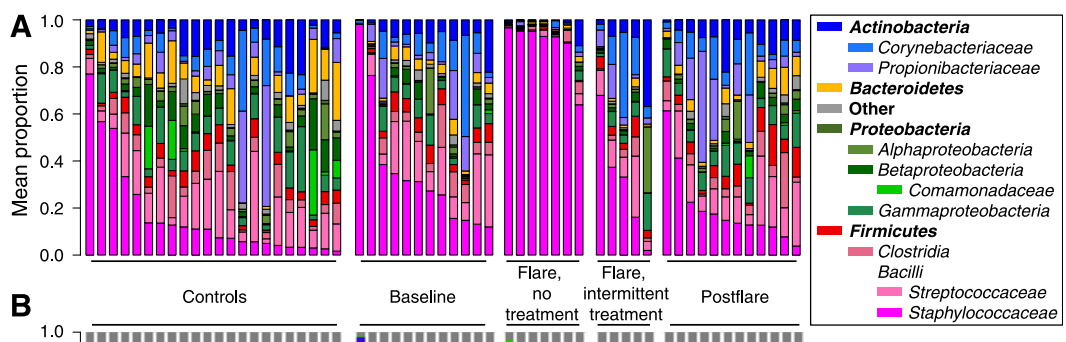

B
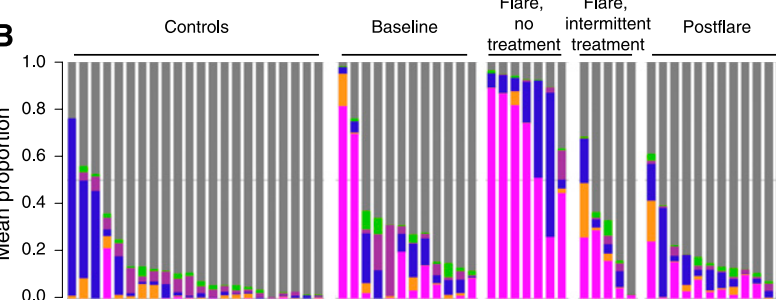

0.0

Figure 3. Bacterial taxonomic classifications in the $A D$ skin microbiome. $(A)$ Mean relative abundance of the 14 major phyla-order in the antecubital (AC) and popliteal creases (PC) for controls and AD disease states: baseline, flare (no-treatment [trt] and intermittent-trt), and postflare (Supplemental Table S13 for order of subjects). (B) Mean relative abundances for Ac and Pc of species-level classifications of staphylococcal species. Order of subjects follows $A$.
Staphylococcaceae

significantly higher than baseline, when some AD patients carry large amounts of $S$. aureus.

With significant shifts in S. aureus abundance observed in AD, we then examined the relationship between the relative abundances of $S$. aureus and Shannon diversity. Adjusting for disease status, the partial correlation between $S$. aureus abundance and Shannon diversity was $r=-0.68, P<6.3 \times 10^{-6}$, indicating that increases in $S$. aureus accounted for reductions in overall diversity observed in this AD cohort (Fig. 4B; Supplemental Table S8).

We then analyzed the relationship between the proportion of $S$. aureus and disease severity. Given the heterogeneity of the microbiome over the human skin surface (Grice et al. 2009), we evaluated the contribution of skin site. Partial correlation between $S$. aureus abundance and disease severity, adjusting for disease state and site, was significant: antecubital $\left(r=0.59, P<2.1 \times 10^{-4}\right)$ and popliteal creases $\left(r=0.57, P<4.3 \times 10^{-4}\right)$ (Fig. 4D; Supplemental Table S9). Volar forearm sites also showed a positive partial correlation $\left(r=0.70, P<1.4 \times 10^{-5}\right)$, possibly related to a field effect, or extension of changes in the microbiome to adjacent skin. This positive partial correlation observed for the volar forearms appeared to be influenced by the increase in body surface area of affected skin extending to the volar forearms during $\mathrm{AD}$ disease flares. The nasal site did not demonstrate a positive correlation. These findings suggest that overabundance of cutaneous $S$. aureus and the associated loss of microbiome diversity are intimately tied to the pathogenesis of AD. Moreover, these data emphasize the regional variation of the skin microbiome at distinct sites and the importance of rational selection of sampling sites in microbiome studies.

Given the dominance of $S$. aureus during AD flares, we sought to determine whether this reduction in diversity was attributed solely to a relative overexpansion of $S$. aureus in the local microbiome. Interestingly, trends in decreased microbial diversity and the similarities of the microbiomes of flaring patients persisted even when the abundance of $S$. aureus sequences was excluded from the analysis and sequence numbers were normalized postremoval (Supplemental Fig. S4; Supplemental Table S10). After subtracting $S$. aureus sequences, we recalculated Shannon and $\theta$ diversity metrics. The decrease in Shannon diversity during the notreatment flares remained significantly different in Ac and Pc $(P<0.05$ flare vs. baseline, postflare, and controls), and was significantly correlated with increasing disease severity (Ac: $r=$ $-0.48, P=0.004$; Pc: $r=-0.44, P=0.009$ ) as compared with the other timepoints and with controls. After removal of $S$. aureus sequences, the no-treatment flares in Ac and Pc remained similar to each other based on Theta index as well. These results suggested that similarities in microbiomes extended beyond $S$. aureus during no-treatment AD flares.

Therefore, in addition to shifts in $S$. aureus, we sought to determine whether the presence of novel bacteria or absence of protective bacteria could play a role in AD flares. While the abundance of $S$. epidermidis in $\mathrm{AD}$ is conflicting in the literature (Gloor et al. 1982; Williams et al. 1990; Higaki et al. 1999), the proportion of $S$. epidermidis during flares was significantly higher than during postflares and 
A
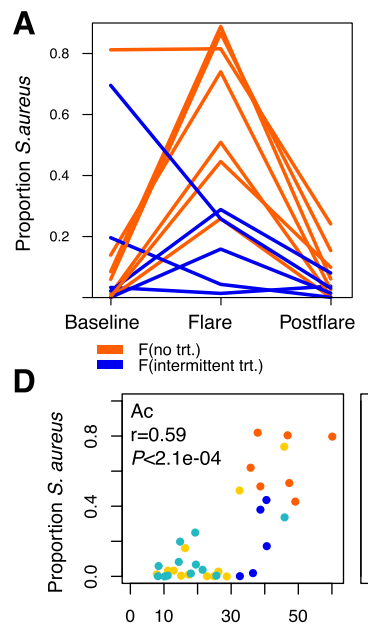

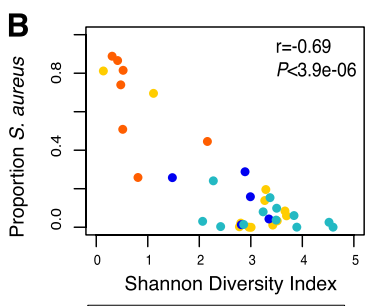
\begin{tabular}{|l|}
\multicolumn{1}{l|}{ Shannon Di } \\
\hline Baseline \\
Flare (no trt.) \\
\hline
\end{tabular} - Flare (int

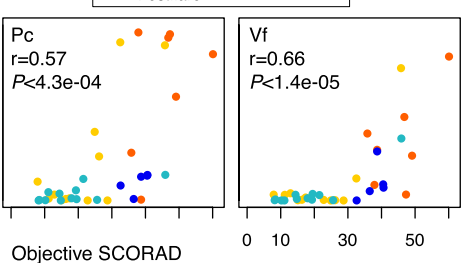

Objective SCORAD

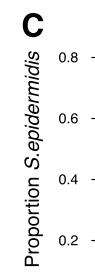
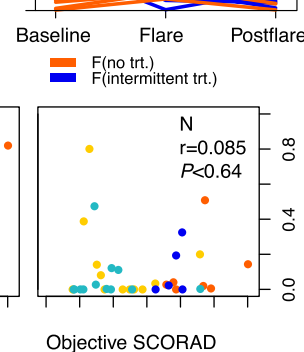

Figure 4. Relationship between staphylococcal species and AD. (A) Longitudinal trend of mean proportion of $S$. aureus in $\mathrm{AD}$ in antecubital and popliteal creases $(\mathrm{AcPc}, n=12)$ grouped by no-treatment (trt) and intermittent-trt flares. (B) Proportion of S. aureus and Shannon diversity index in AcPc. Partial correlation (adjusting for disease state, ACPC). (C) Longitudinal trend of mean proportion of $S$. epidermidis in ACPc. (D) Correlation of proportion of $S$. aureus versus objective SCORAD for each site (AC, $\mathrm{PC}$, Volar forearm/Vf, Nares/N). Partial correlation (adjusting for disease state and site).

in controls ( $P<0.034$ and $P<0.006$, respectively) (Fig 4C). S. epidermidis was also the dominant staphylococcal species in the AcPc of controls (Spearman correlation, $\mathrm{r}=0.98, P<8.9 \times 10^{-8}$; Supplemental Table $\mathrm{S} 7$ ). These findings demonstrate distinct selective shifts among at least two staphylococcal species relative to disease status.

We also identified additional genera over-represented at baseline and postflare. We used the Wilcoxon rank-sum test for comparisons of no-treatment flares with baseline and postflare. We observed a significant relative increase in Streptococcus, Corynebacterium, and Propionibacterium species post-treatment (Supplemental Table S11). With the sequencing depth in this study (Supplemental Fig. S5; Supplemental Table S12) we also identified several lower-abundance bacteria such as the Rothia species, which were also statistically significantly increased between disease timepoints (Supplemental Table S11). To compare the taxa present during baseline and postflare, we used matched-pairs analyses to examine whether the same taxa were abundant, or whether new taxa appeared during postflare. In total, $58 \%$ of taxa (272 of 469) observed at either baseline or postflare were present at both timepoints. Of the taxa present in $>9 / 12$ patients (Supplemental Table S11), all taxa were present at both baseline and postflare, none at statistically significantly different quantities. We also determined that the abundance of genera between baseline and postflare was significantly correlated (partial correlation adjusting for patient, $r=0.61, P<2.2 \times 10^{-16}$ ), indicating that there is a relative expansion of taxa already present in the microbiome with a relatively low number of newly introduced bacteria detected at our level of sampling.

\section{Genomic analyses and culture data comparison}

Since cultivation studies have demonstrated a higher likelihood of $S$. aureus colonization of $\mathrm{AD}$ skin in positive nasal carriers (Williams et al. 1998; Breuer et al. 2002), we examined the results from $S$. aureus cultures concurrently obtained with genomic sampling. Assessment of $S$. aureus nares bioburden (measured as colonies per sample: $0,1-10,>10)$ was significantly associated with relative abundance of $S$. aureus nares genomic sequences $P<0.0072$ ( 0 vs. $1-10$ colonies/ sample); $P<2.1 \times 10^{-6}$ ( 0 vs. $>10$ colonies/ sample) (Supplemental Fig. S6; Supplemental Table S13), suggesting that genomic identification tracked with traditional cultivation methods for this easily cultured microbe. All AD patients had $\geq 1$ nares culture positive for $S$. aureus as compared with $55 \%$ of controls (Table 1 ). Genomic analysis of nares samples did not detect an association between mean objective SCORAD and the relative abundance of $S$. aureus genomic sequences (Fig. 4D; Supplemental Table S9).

In an effort to determine whether a particular $S$. aureus subtype predominated during flares, we examined the microbial population dynamics of unique $S$. aureus $16 \mathrm{~S}$ rRNA sequences. The heterogeneity of the 16S rRNA sequences supports earlier studies demonstrating absence of predominant $S$. aureus clones or biotypes (Hoeger et al. 1992; Lomholt et al. 2005; Kim et al. 2009; Yeung et al. 2011).

\section{Associations with treatment}

Prior studies have shown conflicting results regarding the effect of topical steroids and antimicrobials on the abundance of $S$. aureus (Lever et al. 1988; Stalder et al. 1994; Williams et al. 1998; Ravenscroft et al. 2003; Gong et al. 2006; Hung et al. 2007; Bath-Hextall et al. 2010). One study demonstrated that use of dilute sodium hypochlorite baths did not completely eliminate $S$. aureus, yet resulted in improved clinical outcomes for $\mathrm{AD}$ patients (Huang et al. 2009). Since bacterial diversity in AD skin differed between intermittent-treatment and no-treatment flares, we sought to confirm whether treatment within a defined period of time prior to flares influenced Shannon diversity. We examined samples obtained from four additional $\mathrm{AD}$ patients during disease flares. Other sampling timepoints for these four patients did not fulfill strict predetermined criteria for classification as baseline and flare, and were not included in original analyses. For the patients who used no treatment prior to flare, relative Shannon diversity was low, similar to findings from the no-treatment flare group (Table 2; Supplemental Table S14). In contrast, AD patients who used any treatment prior to flare sampling exhibited the higher diversity characteristic of the intermittent-treatment flare group. One particular $\mathrm{AD}$ patient was sampled during two different flares, but with two different treatment regimens prior to flare sampling. During the first flare, the patient used no topical treatments in the week prior to sampling and demonstrated reduced Shannon diversity (0.626). Before the second flare sampling, the patient used intermittent dilute bleach baths and had a higher Shannon diversity (1.650). Using the Wilcoxon rank-sum test, the mean Shannon diversity for all flares was statistically significantly different between all no-treatment and intermittent-treatment flares (Fig. 5) $(\mathrm{P}<0.0025, n=16)$. These findings suggest that antimicrobial or anti-inflammatory medications decreased $S$. aureus predominance (Supplemental Table S14), affecting bacterial diversity during flares.

\section{Genome Research}




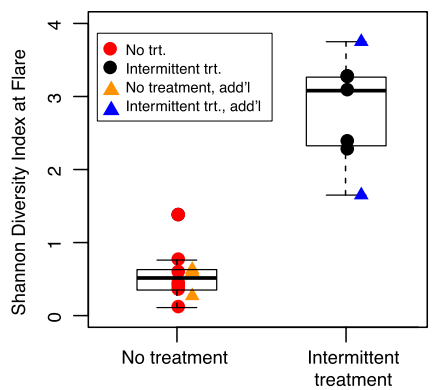

Figure 5. Shannon diversity index during flares. Circles indicate $A D$ patients with a full longitudinal cycle (baseline, flare, and postflare). Triangles indicate flares from four additional (add'l) patients. Means between no-treatment (trt) and intermittent-trt tested with Wilcoxon ranksum test $(P<0.0025, n=16)$.

\section{Discussion}

$\mathrm{AD}$ represents a classic inflammatory skin disorder associated with a specific bacterial species. It is often managed with antimicrobial approaches and as such, AD serves as an example of a disorder in which the contribution of microorganisms to disease course can be examined via metagenomic and microbial community analyses. Using direct 16S rRNA gene sequencing, we defined and characterized the fluctuation of skin bacterial communities during the representative basal (baseline), exacerbation (flare), and resolution (postflare) phases of $\mathrm{AD}$. Our microbiome analyses demonstrated a topographic and temporal shift in the predominant bacterial species and the bacterial diversity in the skin of pediatric $\mathrm{AD}$ patients.

A hallmark of $\mathrm{AD}$ is the chronic, recurrent flaring of intensely itchy skin. By serially analyzing AD patients, we demonstrated a strong association between worsening disease severity and lower skin bacterial diversity. We also determined that this microbiome shift is primarily localized to sites of disease predilection. Because many dermatologic disorders present with a stereotypical body distribution, the site-specificity of skin bacterial communities suggests that not only do particular ecological niches of the skin favor the growth of certain bacteria, but that communities of microbes are also important in the initiation and perpetuation of certain skin diseases.

Direct 16S rRNA gene sequencing of samples obtained during different disease states of $\mathrm{AD}$ provided a less-biased microbiological survey of the selective shifts of Staphylococcus, particularly of $S$. aureus. While $S$. aureus skin colonization and infection is a common feature of AD (Leyden et al. 1974), we have expanded these observations by demonstrating concurrent fluctuations of other skin bacteria. For example, $S$. epidermidis is the predominant Staphylococcus species in the skin of controls and has been considered a commensal in healthy skin (Iwase et al. 2010; Lai et al. 2010), with the ability to inhibit $S$. aureus (Iwase et al. 2010). Although results from previous culture-based studies are conflicting (Gloor et al. 1982; Williams et al. 1990; Higaki et al. 1999), our genomic analysis showed that the proportion of $S$. epidermidis consistently increased during no-treatment flares. The simultaneous prevalence of both $S$. aureus and S. epidermidis that we observed during disease flares provides new insights into the relationship between staphylococci. These staphylococcal species may share a mutualistic or commensal relationship to enhance common resistance to antimicrobial peptides (Peschel et al. 2001; Sieprawska-Lupa et al. 2004; Lai et al. 2007; Li et al. 2007) or enhance binding to exposed extracellular matrix proteins in inflamed AD skin (Nilsson et al. 1998; McCrea et al. 2000; Cho et al. 2001; Williams et al. 2002). Alternatively, the observed con- cordance may represent a compensatory or antagonistic mechanism of $S$. epidermidis, increasing in an attempt to control S. aureus.

In addition to the selective shifts in Staphylococcus species, the relative abundance of Streptococcus, Corynebacterium, and Propionibacterium species varied across the $\mathrm{AD}$ disease states, highlighting the complex relationships among bacteria and the importance of comprehensive investigation of microbial communities. S. aureus and $S$. epidermidis production of antibacterial compounds, including bacteriocins and antimicrobial peptides (Cogen et al. 2010a,b; Iwase et al. 2010; Lai et al. 2010; Joo et al. 2011), may contribute to the relative decrease in Streptococcus, Corynebacterium, and Propionibacterium species observed during AD flares. In addition to identifying changes in the skin commensal population, we determined that low-abundance genera including Rothia species were over-represented during different disease states in all patients. Furthermore, our data suggested that following treatment, increasing diversity of the microbiome, similar to the diversity observed in healthy skin, arises from a relative expansion of taxa already present in the skin microbial community with colonization of few additional new genera not present at baseline. Greater sampling depth in future studies may demonstrate that the majority of taxa present at baseline persist in low numbers during flares, then re-expand following treatment. Overall, the ability to globally examine bacterial community dynamics highlights a major advantage of microbial genomics, defining parameters needed for the development of new methods to maintain or enhance beneficial microbes and microbial diversity to promote health.

While bacterial culture methods are readily implemented and are relatively inexpensive in comparison to $16 \mathrm{~S}$ rRNA gene sequencing, genomic methods are highly complementary, are rapidly dropping in cost and turnaround time, and can identify bacteria that are not easily cultivatable, yet may be important contributors to disease pathophysiology or progression. For easily cultured bacteria such as $S$. aureus, we have shown that the relative abundance of genomic sequences from nasal samples corresponds to the bacterial bioburden assessed using traditional cultivation techniques. However, these results strengthen assertions that genomic analyses of more difficult-to-cultivate bacteria represent the true relative abundance of those bacteria in a sample. No association was identified between the relative abundance of $S$. aureus genomic sequences in nasal samples and disease severity, suggesting that although the nares may serve as a reservoir for $S$. aureus, there may not be a dose-response between the abundance of nasal $S$. aureus and AD severity. Culture-based methods remain important and provide vital information on bioburden. However, the higher resolution and reduced bias of sequence-based and culture-independent microbiome studies in human diseases is of great benefit.

We determined that $\mathrm{AD}$ flares are characterized by low bacterial diversity in the absence of recent treatment. In contrast, intermittent or active treatment is associated with higher bacterial diversity. Although the impact of common AD therapies on $S$. aureus levels has been debated (Stalder et al. 1994; Williams et al. 1998; Ravenscroft et al. 2003), our results and prior studies (Huang et al. 2009) indicate that the clinical effectiveness of AD treatments does not rely on elimination of $S$. aureus. However, AD therapeutic modalities may act to recalibrate the diversity of the skin microbiome. For intermittent-treatment flare patients who used sporadic $\mathrm{AD}$ treatments in the week preceding flare sampling, the early shift toward a diverse microbiome in the presence of active clinical disease suggests that known effective $\mathrm{AD}$ treatments diversify the $\mathrm{AD}$ skin bacterial community. Intermittent-treatment flares likely 
represent a point in the continuum of the disease during transition from flare to postflare. However, the presence of active clinical disease during intermittent-treatment flares suggests that lesional skin requires continued intensive treatment to sufficiently reduce the inflammatory response toward that observed in the postflare state. Increases in diversity associated with AD treatments may be due to therapies that preferentially kill $S$. aureus, allowing relative expansion of other bacteria, promotion of microbes that control $S$. aureus predominance, or total reduction of skin microbes, followed by rapid repopulation with a diverse community.

In summary, specific $\mathrm{AD}$ disease states are characterized by concurrent and anticorrelated shifts in microbial diversity and proportion of Staphylococcus. Since untreated flares have reduced diversity and high Staphylococcus proportions as compared with baseline, postflares, and, notably, intermittent-treatment flares, we propose that increases in the proportion of Staphylococcus and reductions in microbial diversity precede worsening of AD disease severity as observed in no-treatment flares (Fig. 6). Relatively high Staphylococcus proportions observed in a few of the baseline timepoints could represent the pre-flare stage, in which staphylococcal levels increase, yet clinical disease has not begun to dramatically worsen. In contrast, reduction in S. aureus proportions and restoration of microbial diversity was observed in the intermittenttreatment flares, which are characterized as having clinically worsened disease. Based on this, we propose that use of AD treatments modify microbial diversity and proportions of Staphylococcus, but that consistent and continued treatment over a period of time is required to induce the "resolving flare" stage, which transitions into a restoration of full microbial diversity and low population levels of Staphyloccocus, typical of a true postflare.

With public health concerns regarding the development of antibiotic resistance, understanding how current treatments affect bacteria and subsequent disease activity will allow us to identify and develop directed therapies for $\mathrm{AD}$ that modify the skin microbiome, potentially reducing use of systemic antibiotics. Our findings highlight the importance of performing longitudinal microbiome studies to examine the influence of microbial communities on health and disease. Advances in technologies including whole-genome shotgun sequencing will continue to enhance our ability to probe microbial communities via metagenomics and functional metabolomics. The ability to interrogate microbial communities en toto and in situ has begun to provide insights regarding the important and diverse roles of microbes in human disorders and how shifts in microbial communities may improve human health.

\section{Methods}

\section{Study design}

A prospective natural history study was approved by the institutional review board of NHGRI, NIH. The study was designed

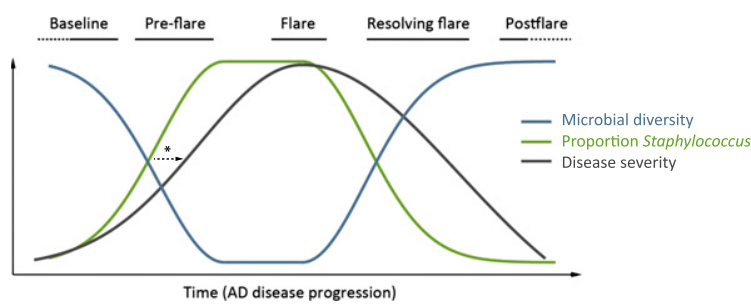

Figure 6. AD microbiome progression hypothesis. $\left(^{*}\right)$ Proposed relationship among shifts in skin microbial diversity, the proportion of Staphylococcus, and disease severity. to evaluate pediatric $\mathrm{AD}$ patients at three timepoints (baseline, flare, and postflare) to capture clinically distinct timepoints that characterize this chronic relapsing, remitting skin disease. Agematched children were sampled at similar time intervals to serve as healthy pediatric controls.

Inclusion criteria for $\mathrm{AD}$ patients included ages 2-18 yr, moderate-to-severe disease, presence of more than or equal to one affected antecubital or popliteal crease at enrollment, and ability to tolerate $>7 \mathrm{~d}$ without topical AD treatments and $>4 \mathrm{wk}$ off both systemic antibiotics and corticosteroids. The diagnosis of AD was based on the UK Working Party definition (Williams et al. 1994a). Objective SCORAD as assessed by one individual (HHK) was used to determine study eligibility and disease status at each clinic visit (Williams et al. 1994b; Kunz et al. 1997; Oranje et al. 2007). Moderate-to-severe disease was defined by objective SCORAD $\geq 15$ (range 0-83) (Oranje et al. 2007).

Exclusion criteria for all subjects included receiving investigational new treatments, ultraviolet light therapy, monoclonal antibodies, systemic immunosuppressants within $7 \mathrm{~d}$ or five half-lives (taking the longer time period) of skin sampling; a history of bone-marrow transplant or gene therapy; and clinically apparent underlying immunodeficiency. Exclusion criteria specific for AD patients included systemic antibiotics during the preceding 2 wk (except for post-flare timepoint). Exclusion criteria for healthy controls also included current or prior chronic skin disorders such as $\mathrm{AD}$ or psoriasis; asthma and allergic rhinitis, via International Study of Asthma and Allergies in Childhood questionnaire (Asher et al. 1995); other chronic medical conditions; and use of systemic antibiotics in the preceding 6 mo.

For AD patients, baseline was defined as usual and stable disease state and ability to tolerate $>7 \mathrm{~d}$ without topical AD treatments to intended sample sites and $>4$ wk off both oral antibiotics and corticosteroids. Flare was defined as acute exacerbation of disease on any skin region prior to initiation of intensified AD treatment and without restriction of usual treatments $>24 \mathrm{~h}$ prior to sampling. Subjects were instructed to promptly contact the research team when skin disease worsening was apparent. Flares were evaluated and sampled within $24 \mathrm{~h}$ of notification to avoid delay in intensified treatment. Postflare was defined as 10-14 d after the initiation of intensified AD treatment. Intensified $\mathrm{AD}$ treatment recommendations could include the following based on their typical regimen: dilute bleach baths ( 0.25 cup of $6 \%$ bleach into bath half filled with water for a final concentration of $0.0005 \%$ ) three to four times per week, higher potency topical steroids twice daily, bland emollients twice daily, and, in certain cases of suspected clinical infection, systemic antibiotics prescribed by primary care physicians (Supplemental Table S1).

\section{Subjects}

AD patients aged $2-15 \mathrm{yr}$ of age, and similarly aged healthy controls were recruited from the larger Washington, DC metropolitan region. Written informed consent was obtained from parents or guardians of all participating children. Complete medical and medication history and skin examination was performed at all clinic visits.

\section{Sample collection}

Skin preparation instructions included avoiding bathing and application of topical medications and emollients for $24 \mathrm{~h}$ at sampling sites prior to all sampling timepoints (baseline, flare, and postflare). Samples were obtained as described (Grice et al. 2009). Nasal cultures were obtained at each timepoint. Blood was drawn during the first visit.

\section{Genome Research}

www.genome.org 


\section{Cultures}

Nasal cultures were performed by the Department of Laboratory Medicine (DLM), Clinical Center, NIH. Cultures were obtained with swabs (BBLtm CultureSwabTM, made by Copan for Becton, Dickinson and Company) and plated on three different growth mediums (Remel Products). After incubation, the colonies were counted and identified on the basis of gross and microscopic assessment after treatment with Gram's stain. Culture results were grouped into 0, 1-10, and >10 colonies of Staphylococcus aureus per sample.

\section{DNA extraction and sequencing}

Skin swabs were incubated in enzymatic lysis buffer and lysozyme $(20 \mathrm{mg} / \mathrm{mL})$ for $30 \mathrm{~min}$ at $37^{\circ} \mathrm{C}$. Two $5-\mathrm{mm}$ stainless steel beads (Qiagen) were added to the solution, placed in a Tissuelyser (Qiagen), and processed for $2 \mathrm{~min}$ at $30 \mathrm{~Hz}$.The standard protocol for the PureLink Genomic DNA kit (Invitrogen) was followed for all subsequent steps. rRNA genes $(16 S)$ were amplified from purified genomic DNA using primers 8F (5'-AGAGTTTGATCCTGGCT CAG-3') and 1391R (5'-GACGGGCGGTGWGTRCA-3'). PCR amplification, cloning, and sequencing were performed as described (Grice et al. 2009). Sequence assembly, alignment, and chimera elimination were performed as described, with specified modifications (Grice et al. 2009; Schloss et al. 2009; Huse et al. 2010).

\section{Analysis pipeline}

\section{Community analysis}

Operational taxonomic units (OTUs) were identified and a distance matrix calculated using mothur software v14.0 (Schloss et al. 2009). No lane masking was applied. The distance matrix was then analyzed to calculate OTUs using the average neighbor algorithm. Community evenness and richness (Shannon diversity index "shannon"), shared community membership, and shared community structure (theta index "thetayc") at a 98\% similarity cutoff were calculated using summary.shared() and summary. single() in mothur. Shannon diversity was calculated as follows: $H_{\text {shannon }}=-\sum_{i=1}^{S_{o b s}} \frac{n_{i}}{N} \ln \frac{n_{i}}{N}$, where $S_{o b s}=$ number of observed OTUs, $n_{i}=$ number of individuals in OTU $i$, and $N=$ total number of individuals in the community. The theta index, or Yue and Clayton measure of similarity between two community structures (Yue and Clayton 2005), was calculated as follows:

$$
C_{\theta_{Y C}}=\frac{\sum_{i=1}^{S_{T}} a_{i} b_{i}}{\sum_{i=1}^{S_{T}}\left(a_{i}-b_{i}\right)^{2}+\sum_{i=1}^{S_{T}} a_{i} b_{i}},
$$

where $S_{T}=$ total number of OTUs in communities A and B, $a_{i}=$ relative abundance of OTU $i$ in community $\mathrm{A}$, and $b_{i}=$ relative abundance of OTU $i$ in community B. To calculate alpha and beta diversity metrics minus $S$. aureus, we removed sequences classified as $S$. aureus from the analysis. Resulting samples were then subsampled $100 \times$ to the lowest number of sequences per group $(\mathrm{Ac}=129, \mathrm{Pc}=$ $31, N=68, \mathrm{Vf}=133$ ) and subsampled results were averaged. Results without subsampling yielded similar results (data not shown).

Because left and right symmetric sites generally exhibit low variability (Grice et al. 2009), the sites were grouped prior to analysis. For timepoint categories baseline, no-treatment flare, intermittenttreatment flare, and control calculations of Shannon diversity and theta indices, values were averaged for the antecubital and popliteal crease unless otherwise indicated. To estimate depth of sequence sampling, rarefaction curve data for each individual at each site was calculated using rarefaction.single in mothur, and individuals for each group were averaged to obtain group-wide rarefaction curves.

\section{Genus-level analysis}

Following sequence trimming, alignment, and other pre-processing steps as previously described, 16S rRNA sequences were classified to the genus level using the Ribosomal Database Project (RDP) classifications v1.0, training set 4 (Cole et al. 2009). For 14 major order-genera that represented $>1 \%$ of total $16 \mathrm{~S}$ rRNA sequences, relative abundances were computed by dividing sequence counts by the total sequences obtained for that site, with proportions for antecubital and popliteal crease averaged. Finally, custom scripts were generated for taxonomic classifications to the species level for staphylococcal species. Staphylococcal sequences were speciated by alignment to a curated collection of staphylococcal reference sequences from complete genome sequences and type strains. Each sequence was assigned a label based on the consensus call of sequence alignments with the lowest edit distance between a query and reference.

\section{Statistics}

All data are represented as mean \pm SEM unless otherwise indicated. For controls with $>1$ timepoint, the relevant data was averaged for each control. As disease severity differed minimally from left to right symmetric sites, left and right values were averaged prior to statistical comparisons. AcPc indicates the mean of values obtained from the antecubital and popliteal crease for each individual (post-averaging of left and right symmetric sites, and for controls, also post-averaging of timepoints).

Analyses involved calculating partial correlations by using the relevant variables for each subject over all timepoints. Calculations were performed on Fisher-transformed $r$ values. For statistical testing of comparisons of differences between groups, the nonparametric Wilcoxon rank sum test was used for all comparisons (wilcox.test in the statistical software program R). Where indicated, within-subjects analysis was performed by using the option "paired = $\mathrm{T}^{\prime \prime}$ in wilcox.test. All $P$-values were adjusted using p.adjust in R using Bonferroni (\# comparisons $\leq 10$ ) or false discovery rate (\# comparisons $>10$ ) corrections. For testing of significantly over-represented genera between the baseline, flare, and post-flare groups, we reduced the number of multiple comparisons by prefiltering the search to the seven genera that were present in $100 \%$ of baseline or no-treatment flares (for baseline-flare comparisons), or the 12 genera that were present in $100 \%$ of no-treatment flares or postflare (for flarepostflare comparisons) prior to performing a paired Wilcoxon rank sum test. For comparisons between baseline and postflare, we limited the search to genera that were present in $>75 \%$ of individuals and performed a paired Wilcoxon rank sum test.

\section{Data access}

The sequence data from this study have been submitted to GenBank (http://www.ncbi.nlm.nih.gov/genbank) and can be accessed through BioProject ID 46333. Patient and sample metadata have been deposited in the controlled access database dbGaP under study accession phs000266.v1.p1.

\section{NISC Comparative Sequencing Program investigators}

Jim Mullikin, Jim Thomas, Robert Blakesley, Alice Young, Grace $\mathrm{Chu}$, Colleen Ramsahoye, Sean Lovett, Joel Han, Richelle Legaspi, Christina Sison, Casandra Montemayor, Michael Gregory, April Hargrove, Taccara Johnson, Nancy Riebow, Brian Schmidt, Betsy Novotny, Jyoti Gupta, Betty Benjamin, Shelise Brooks, Holly Coleman, Shi-ling Ho, Karen Schandler, Mal Stantripop, Quino Maduro, 
Gerry Bouffard, Mila Dekhtyar, Xiaobin Guan, Cathy Masiello, Baishali Maskeri, Jenny McDowell, Morgan Park, and Meg Vemulapalli.

\section{Acknowledgments}

We thank Mark C. Udey, Eric Green, and Evan Snitkin for helpful discussions; Pamela Thomas, Deborah Schoenfeld, Joie Davis, Roselyn Epps, Celeste Nelson, and Donna Gaskins for underlying contributions; and especially the patients and volunteers. This work was supported by NIH CC, NCI, and NHGRI Intramural Research Programs, and in part by 1K99AR059222 (H.H.K.). Sequencing and clinical research support was funded by grants from the National Institutes of Health Common Fund Human Microbiome Project (1UH2AR057504-01 and 4UH3AR057504-02).

\section{References}

Archer GL, Climo MW. 2001. Staphylococcus aureus bacteremia-consider the source. N Engl J Med 344: 55-56.

Asher MI, Keil U, Anderson HR, Beasley R, Crane J, Martinez F, Mitchell EA, Pearce N, Sibbald B, Stewart AW, et al. 1995. International Study of Asthma and Allergies in Childhood (ISAAC): rationale and methods. Eur Respir J 8: 483-491.

Asher MI, Montefort S, Bjorksten B, Lai CK, Strachan DP, Weiland SK, Williams H. 2006. Worldwide time trends in the prevalence of symptoms of asthma, allergic rhinoconjunctivitis, and eczema in childhood: ISAAC phases one and three repeat multicountry crosssectional surveys. Lancet 368: 733-743.

Bath-Hextall FJ, Birnie AJ, Ravenscroft JC, Williams HC. 2010. Interventions to reduce Staphylococcus aureus in the management of atopic eczema: an updated Cochrane review. Br J Dermatol 163: 12-26.

Bickers DR, Lim HW, Margolis D, Weinstock MA, Goodman C, Faulkner E, Gould C, Gemmen E, Dall T. 2006. The burden of skin diseases: 2004: A joint project of the American Academy of Dermatology Association and the Society for Investigative Dermatology. J Am Acad Dermatol 55: 490-500.

Breuer K, HÄussler S, Kapp A, Werfel T. 2002. Staphylococcus aureus: colonizing features and influence of an antibacterial treatment in adults with atopic dermatitis. Br J Dermatol 147: 55-61.

Cho SH, Strickland I, Boguniewicz M, Leung DY. 2001. Fibronectin and fibrinogen contribute to the enhanced binding of Staphylococcus aureus to atopic skin. J Allergy Clin Immunol 108: 269-274.

Cogen AL, Yamasaki K, Muto J, Sanchez KM, Crotty Alexander L, Tanios J, Lai Y, Kim JE, Nizet V, Gallo RL. 2010a. Staphylococcus epidermidis antimicrobial $\delta$-toxin (phenol-soluble modulin- $\gamma$ ) cooperates with host antimicrobial peptides to kill group A Streptococcus. PLoS ONE 5: e8557. doi: 10.1371/journal.pone.0008557.

Cogen AL, Yamasaki K, Sanchez KM, Dorschner RA, Lai Y, MacLeod DT, Torpey JW, Otto M, Nizet V, Kim JE, et al. 2010b. Selective antimicrobial action is provided by phenol-soluble modulins derived from Staphylococcus epidermidis, a normal resident of the skin. J Invest Dermatol 130: $192-200$

Cole JR, Wang Q, Cardenas E, Fish J, Chai B, Farris RJ, Kulam-Syed-Mohideen AS, McGarrell DM, Marsh T, Garrity GM, et al. 2009. The Ribosomal Database Project: improved alignments and new tools for rRNA analysis. Nucleic Acids Res 37: D141-D145.

Costello EK, Lauber CL, Hamady M, Fierer N, Gordon JI, Knight R. 2009. Bacterial community variation in human body habitats across space and time. Science 326: 1694-1697.

Cramer C, Link E, Horster M, Koletzko S, Bauer CP, Berdel D, von Berg A Lehmann I, Herbarth O, Borte M, et al. 2010. Elder siblings enhance the effect of filaggrin mutations on childhood eczema: results from the 2 birth cohort studies LISAplus and GINIplus. J Allergy Clin Immunol 125: 1254-1260.

Gao Z, Tseng CH, Pei Z, Blaser MJ. 2007. Molecular analysis of human forearm superficial skin bacterial biota. Proc Natl Acad Sci 104: 29272932.

Gloor M, Peters G, Stoika D. 1982. On the resident aerobic bacterial skin flora in unaffected skin of patients with atopic dermatitis and in healthy controls. Dermatologica 164: 258-265.

Gong JQ, Lin L, Lin T, Hao F, Zeng FQ, Bi ZG, Yi D, Zhao B. 2006. Skin colonization by Staphylococcus aureus in patients with eczema and atopic dermatitis and relevant combined topical therapy: a double-blind multicentre randomized controlled trial. Br J Dermatol 155: 680-687.
Grice EA, Kong HH, Conlan S, Deming CB, Davis J, Young AC, Bouffard GG Blakesley RW, Murray PR, Green ED, et al. 2009. Topographical and temporal diversity of the human skin microbiome. Science 324: 11901192 .

Hidron AI, Edwards JR, Patel J, Horan TC, Sievert DM, Pollock DA, Fridkin SK. 2008. NHSN annual update: antimicrobial-resistant pathogens associated with healthcare-associated infections: annual summary of data reported to the National Healthcare Safety Network at the Centers for Disease Control and Prevention, 2006-2007. Infect Control Hosp Epidemiol 29: 996-1011.

Higaki S, Morohashi M, Yamagishi T, Hasegawa Y. 1999. Comparative study of staphylococci from the skin of atopic dermatitis patients and from healthy subjects. Int J Dermatol 38: 265-269.

Hoeger PH, Lenz W, Boutonnier A, Fournier JM. 1992. Staphylococcal skin colonization in children with atopic dermatitis: prevalence, persistence, and transmission of toxigenic and nontoxigenic strains. J Infect Dis 165: 1064-1068.

Huang JT, Abrams M, Tlougan B, Rademaker A, Paller AS. 2009. Treatment of Staphylococcus aureus colonization in atopic dermatitis decreases disease severity. Pediatrics 123: e808-e814.

Hung SH, Lin YT, Chu CY, Lee CC, Liang TC, Yang YH, Wang LC, Chiang BL. 2007. Staphylococcus colonization in atopic dermatitis treated with fluticasone or tacrolimus with or without antibiotics. Ann Allergy Asthma Immunol 98: 51-56.

Huse SM, Welch DM, Morrison HG, Sogin ML. 2010. Ironing out the wrinkles in the rare biosphere through improved OTU clustering. Environ Microbiol 12: 1889-1898.

Iwase T, Uehara Y, Shinji H, Tajima A, Seo H, Takada K, Agata T, Mizunoe Y. 2010. Staphylococcus epidermidis Esp inhibits Staphylococcus aureus biofilm formation and nasal colonization. Nature 465: 346-349.

Joo HS, Cheung GY, Otto M. 2011. Antimicrobial activity of communityassociated methicillin-resistant Staphylococcus aureus is caused by phenol-soluble modulin derivatives. J Biol Chem 286: 8933-8940.

Kim DW, Park JY, Park KD, Kim TH, Lee WJ, Lee SJ, Kim J. 2009. Are there predominant strains and toxins of Staphylococcus aureus in atopic dermatitis patients? Genotypic characterization and toxin determination of $S$. aureus isolated in adolescent and adult patients with atopic dermatitis. J Dermatol 36: 75-81.

Kunz B, Oranje AP, Labreze L, Stalder JF, Ring J, Taieb A. 1997. Clinical validation and guidelines for the SCORAD index: consensus report of the European Task Force on Atopic Dermatitis. Dermatology 195: 10-19.

Lai Y, Villaruz AE, Li M, Cha DJ, Sturdevant DE, Otto M. 2007. The human anionic antimicrobial peptide dermcidin induces proteolytic defence mechanisms in staphylococci. Mol Microbiol 63: 497-506.

Lai Y, Cogen AL, Radek KA, Park HJ, Macleod DT, Leichtle A, Ryan AF, Di Nardo A, Gallo RL. 2010. Activation of TLR2 by a small molecule produced by Staphylococcus epidermidis increases antimicrobial defense against bacterial skin infections. J Invest Dermatol 130: 22112221.

Lever R, Hadley K, Downey D, Mackie R. 1988. Staphylococcal colonization in atopic dermatitis and the effect of topical mupirocin therapy. $\mathrm{Br} J$ Dermatol 119: 189-198.

Leyden JJ, Marples RR, Kligman AM. 1974. Staphylococcus aureus in the lesions of atopic dermatitis. Br I Dermatol 90: 525-530.

Li M, Lai Y, Villaruz AE, Cha DJ, Sturdevant DE, Otto M. 2007. Gram-positive three-component antimicrobial peptide-sensing system. Proc Natl Acad Sci 104: 9469-9474.

Lomholt H, Andersen KE, Kilian M. 2005. Staphylococcus aureus clonal dynamics and virulence factors in children with atopic dermatitis. Invest Dermatol 125: 977-982.

McCrea KW, Hartford O, Davis S, Eidhin DN, Lina G, Speziale P, Foster TJ, Hook M. 2000. The serine-aspartate repeat (Sdr) protein family in Staphylococcus epidermidis. Microbiology 146: 1535-1546.

Nilsson M, Frykberg L, Flock JI, Pei L, Lindberg M, Guss B. 1998. A fibrinogen-binding protein of Staphylococcus epidermidis. Infect Immun 66: 2666-2673.

Ong PY, Ohtake T, Brandt C, Strickland I, Boguniewicz M, Ganz T, Gallo RL, Leung DY. 2002. Endogenous antimicrobial peptides and skin infections in atopic dermatitis. N Engl J Med 347: 1151-1160.

Oranje AP, Glazenburg EJ, Wolkerstorfer A, de Waard-van der Spek FB. 2007 Practical issues on interpretation of scoring atopic dermatitis: the SCORAD index, objective SCORAD and the three-item severity score. $\mathrm{Br}$ J Dermatol 157: 645-648.

Palmer CN, Irvine AD, Terron-Kwiatkowski A, Zhao Y, Liao H, Lee SP, Goudie DR, Sandilands A, Campbell LE, Smith FJ, et al. 2006. Common loss-of-function variants of the epidermal barrier protein filaggrin are a major predisposing factor for atopic dermatitis. Nat Genet $\mathbf{3 8}$ : $441-446$

Peschel A, Jack RW, Otto M, Collins LV, Staubitz P, Nicholson G, Kalbacher $\mathrm{H}$, Nieuwenhuizen WF, Jung G, Tarkowski A, et al. 2001. Staphylococcus aureus resistance to human defensins and evasion of neutrophil killing

\section{Genome Research}


via the novel virulence factor MprF is based on modification of membrane lipids with l-lysine. J Exp Med 193: 1067-1076.

Ravenscroft JC, Layton AM, Eady EA, Murtagh MS, Coates P, Walker M, Cove JH. 2003. Short-term effects of topical fusidic acid or mupirocin on the prevalence of fusidic acid resistant (FusR) Staphylococcus aureus in atopic eczema. Br J Dermatol 148: 1010-1017.

Sandilands A, Terron-Kwiatkowski A, Hull PR, O'Regan GM, Clayton TH, Watson RM, Carrick T, Evans AT, Liao H, Zhao Y, et al. 2007. Comprehensive analysis of the gene encoding filaggrin uncovers prevalent and rare mutations in ichthyosis vulgaris and atopic eczema. Nat Genet 39: 650-654.

Schloss PD, Handelsman J. 2006. Introducing SONS, a tool for operational taxonomic unit-based comparisons of microbial community memberships and structures. Appl Environ Microbiol 72: 6773-6779.

Schloss PD, Westcott SL, Ryabin T, Hall JR, Hartmann M, Hollister EB, Lesniewski RA, Oakley BB, Parks DH, Robinson CJ, et al. 2009. Introducing mothur: open-source, platform-independent, communitysupported software for describing and comparing microbial communities. Appl Environ Microbiol 75: 7537-7541.

Shaw TE, Currie GP, Koudelka CW, Simpson EL. 2011. Eczema prevalence in the United States: data from the 2003 National Survey of Children's Health. J Invest Dermatol 131: 67-73.

Sieprawska-Lupa M, Mydel P, Krawczyk K, Wojcik K, Puklo M, Lupa B, Suder P, Silberring J, Reed M, Pohl J, et al. 2004. Degradation of human antimicrobial peptide LL-37 by Staphylococcus aureus-derived proteinases. Antimicrob Agents Chemother 48: 4673-4679.

Stalder JF, Fleury M, Sourisse M, Rostin M, Pheline F, Litoux P. 1994. Local steroid therapy and bacterial skin flora in atopic dermatitis. Br J Dermatol 131: 536-540.

von Eiff C, Becker K, Machka K, Stammer H, Peters G. 2001. Nasal carriage as a source of Staphylococcus aureus bacteremia. Study Group. N Engl J Med 344: 11-16. von Mutius E. 2000. The environmental predictors of allergic disease. J Allergy Clin Immunol 105: 9-19.

Williams RE, Gibson AG, Aitchison TC, Lever R, Mackie RM. 1990. Assessment of a contact-plate sampling technique and subsequent quantitative bacterial studies in atopic dermatitis. Br J Dermatol 123: 493-501.

Williams HC, Burney PG, Pembroke AC, Hay RJ. 1994a. The U.K. working party's diagnostic criteria for atopic dermatitis. III. Independent hospital validation. Br J Dermatol 131: 406-416.

Williams HC, Burney PG, Strachan D, Hay RJ. 1994b. The U.K. working party's diagnostic criteria for atopic dermatitis. II. Observer variation of clinical diagnosis and signs of atopic dermatitis. Br J Dermatol 131: 397-405.

Williams JV, Vowels BR, Honig PJ, Leyden JJ. 1998. S. aureus isolation from the lesions, the hands, and the anterior nares of patients with atopic dermatitis. Pediatr Dermatol 15: 194-198.

Williams RJ, Henderson B, Sharp LJ, Nair SP. 2002. Identification of a fibronectin-binding protein from Staphylococcus epidermidis. Infect Immun 70: 6805-6810.

Wisplinghoff H, Bischoff T, Tallent SM, Seifert H, Wenzel RP, Edmond MB. 2004. Nosocomial bloodstream infections in US hospitals: analysis of 24,179 cases from a prospective nationwide surveillance study. Clin Infect Dis 39: 309-317.

Yeung M, Balma-Mena A, Shear N, Simor A, Pope E, Walsh S, McGavin MJ. 2011. Identification of major clonal complexes and toxin producing strains among Staphylococcus aureus associated with atopic dermatitis. Microbes Infect 13: 189-197.

Yue JC, Clayton MK. 2005. A similarity measure based on species proportions. Comm Statist Theory Methods 34: 2123-2131.

Received August 23, 2011; accepted in revised form January 30, 2012. 


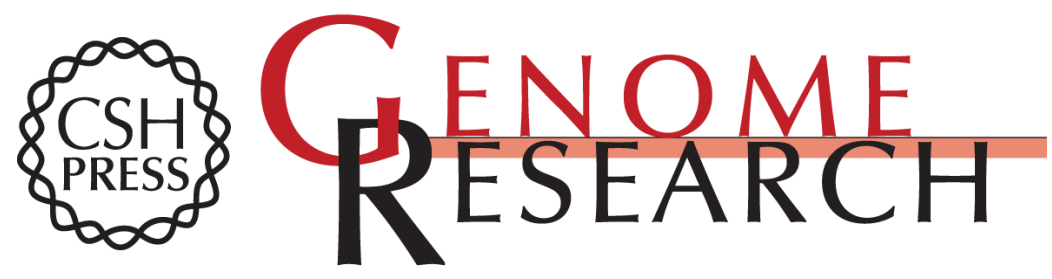

\section{Temporal shifts in the skin microbiome associated with disease flares and treatment in children with atopic dermatitis}

Heidi H. Kong, Julia Oh, Clay Deming, et al.

Genome Res. 2012 22: 850-859 originally published online February 6, 2012

Access the most recent version at doi:10.1101/gr.131029.111

Supplemental Material

References

Creative

Commons

License

Email Alerting Service
http://genome.cshlp.org/content/suppl/2012/02/06/gr.131029.111.DC1

This article cites 55 articles, 12 of which can be accessed free at: http://genome.cshlp.org/content/22/5/850.full.html\#ref-list-1

This article is distributed exclusively by Cold Spring Harbor Laboratory Press for the first six months after the full-issue publication date (see

$\mathrm{http}: / / g$ enome.cshlp.org/site/misc/terms.xhtml). After six months, it is available under a Creative Commons License (Attribution-NonCommercial 3.0 Unported License), as described at http://creativecommons.org/licenses/by-nc/3.0/.

Receive free email alerts when new articles cite this article - sign up in the box at the top right corner of the article or click here.

\section{Affordable, Accurate Sequencing.}

To subscribe to Genome Research go to:

https://genome.cshlp.org/subscriptions 